\title{
Methods and Architectural Patterns of Storage, Analysis and Distribution of Spatio-temporal Data
}

\author{
Stanislav A. Yamashkin ${ }^{1}$ \\ Institute of Electronic and Lighting Engineering \\ National Research Mordovia State University \\ Saransk, Russia
}

\author{
Anatoliy A. Yamashkin ${ }^{2}$ \\ Geography Faculty \\ National Research Mordovia State University \\ Saransk, Russia
}

\author{
Ekaterina O. Yamashkina ${ }^{3}$, Sergey M. Kovalenko ${ }^{4}$ \\ Institute of Information Technologies, MIREA — Russian Technological University \\ Moscow, Russia
}

\begin{abstract}
The work describes the key principles of the process of building digital spatial data infrastructures for effective decision-making in the management of natural systems and for the sustainable development of the regional economy. The following reference points are considered in detail: increasing the accuracy of the deep learning and neural networks algorithmic and software for the process of analyzing spatial data, developing storage systems for large spatio-temporal data by developing new physical and logical storage models, introducing effective geoportal technologies and developing new architectural patterns for presentation and further dissemination of spatio-temporal using modern web technologies. The plan for working out a scientific problem of development of methods and architectural patterns of storage, analysis and distribution of spatio-temporal data determined the structure of the article. The first section concretizes the criteria of efficiency of information processes in the digital spatial data infrastructure (SDI), the second section discusses algorithmic support of the process of analysis of spatial data, the third - integration of spatial data, and finally, the final section - implementation and projectoriented use of geoportal systems.
\end{abstract}

Keywords-Spatial data infrastructure; deep learning; neural networks; spatial data; geoportals

\section{INTRODUCTION}

SDI (Spatial Data Infrastructure) is an essential element in addressing the fundamental problem of efficient analysis, systematization and use of large volumes of heterogeneous spatial data, their visualization and dissemination for management decisions on optimizing territorial systems in the context of sustainable development of countries and regions.

The scientific problem of the formation of the theory and methodology for the development of digital technologies in the field of sustainable development, which makes it possible to increase the efficiency of economic activity, prevent and minimize the consequences of natural and natural-man-made environmental emergencies in the context of global climate change, is an urgent challenge of our time.

The development of digital spatial data infrastructures (SDI) should be based on a comprehensive solution to a system of problems aimed at finding effective solutions in the field of integration, processing and analysis, storage, visualization and dissemination of large volumes of spatial and temporal data:

1) Creation and experimental substantiation of new geoinformation methods and algorithms for complex interpretation of remote sensing data, digital maps and auxiliary space-time information using deep machine learning.

2) An experimental study of systemic connections and patterns of functioning and development of natural-socialproduction systems (NSPS) with the subsequent development of new high-precision algorithms for predicting the development of spatio-temporal processes.

3) Development of a system of methods for integrating knowledge, storing and processing large arrays of spatiotemporal data in digital SDI using cloud technologies, revealing the aspects of effective consolidation, operational analysis and synthesis of relevant data for the purpose of their further use in making management decisions.

4) Creation of effective architectural solutions in the field of building geoportal systems for the integration of knowledge, visualization and dissemination of spatio-temporal data of digital SDI in order to organize a fundamental basis for the synthesis and system analysis of expert information when making management decisions in the field of sustainable development, forecasting the development of natural and manmade processes, including natural phenomena.

The introduction of SDI allows you to approach the solution of the problem of consolidating spatial information for making science-based management decisions. For this geoportal systems required for high-precision automated monitoring of geotechnical systems and for anticipating the consequences of environmental emergencies offer promising avenues for development. SDIs combine software and hardware tools for processing, storing, presenting and delivering geospatial data to end-users to address scientific and applied issues. The visualization, dissemination and use of spatial data infrastructures necessarily address the digital economy. 
The plan for working out a scientific problem determined the structure of the article. The first section concretizes the criteria of efficiency of information processes in the digital SDI, the second section discusses algorithmic support of the process of analysis of spatial data, the third - integration of spatial data, and finally, the final section - implementation and project-oriented use of geoportal systems.

\section{CRITERIA OF EFFICIENCY OF INFORMATION PROCESSES IN THE DIGITAL SDI}

SDIs should integrate the following components for analysis, storage and dissemination of geospatial data connected through interfaces.

1) The subsystem of analysis (SA) of large spatial data is used to study and update stored information for effective decision-making within the project.

From the point of view of the potential for attainability of target effects, the following formalized criteria play an important role: increasing of objective metrics $\left(E_{A 1}\right)$, reduction of type I and II errors $\left(E_{A 2}\right)$, optimization of expert assessments $\left(E_{A 3}\right)$. When assessing the resource intensity of performing operations for analyzing spatial data, the following are key: indicators of algorithms optimization $\left(\mathfrak{R}_{A I}\right)$ and hardware power $\left(\mathfrak{R}_{A 2}\right)$. The complexity of the processes of creating, modifying and using the process analysis subsystem can be determined by the modularity of the model $\left(T_{A I}\right)$, optimization of software metrics $\left(T_{A 2}\right)$, testability indicators $\left(T_{A 3}\right)$.

The solution to the problem of improving the accuracy and speed of the methodological, algorithmic and software for the process of analyzing spatial data and predicting space-time processes is possible through the development and use of new algorithms for deep machine analysis for the complex interpretation of remote sensing data, digital synthetic landscape maps and auxiliary space-time information in order to implementation of high-precision automated monitoring of the state of ecological-socio-economic systems and forecasting of natural and man-made emergencies.

2) Spatial data storage centers (SDSC) play the role of a system component of the SDI used to provide storage and retrieval of relevant spatial data.

The achievability of target effects is determined by the complexity and completeness $\left(E_{S 1}\right)$, relevance, reliability and usefulness $\left(E_{S 2}\right)$, security $\left(E_{S 3}\right)$ of spatial data. The resource intensity of design-oriented digital SDIs in the implementation of information processes is based on the indexing of records $\left(\Re_{S 1}\right)$, optimization and caching of requests $\left(\mathfrak{R}_{S 2}\right)$, development of search algorithms $\left(\mathfrak{R}_{S 3}\right)$. The complexity of the processes of creating, modifying and using centers for storing spatial data is based on standardization and documentation $\left(T_{S 1}\right)$, integrity $\left(T_{S 2}\right)$, database normalization $\left(T_{S 3}\right)$.
Improvement of storage systems for large spatio-temporal data is possible through the development of new physical and logical storage models, synthesis of relational and NoSQL approaches, the introduction of new algorithms for caching and indexing large information arrays, the creation of methods for storing and processing large amounts of information using cloud technologies.

3) Geoportals are the external component of the system. They play the role of an access point to actual spatial data through search and visualization tools.

The achievability potential of the target effects of geoportals is based on indicators of functional suitability and project orientation $\left(E_{G 1}\right)$, convenience and reliability of use $\left(E_{G 2}\right)$, security of user data $\left(E_{G 3}\right)$; resource intensity when performing operations for obtaining relevant spatial information - based on the indicators of software optimization of the geoportal framework $\left(\mathfrak{R}_{G 1}\right)$, increasing the resources of the dedicated server $\left(\mathfrak{R}_{G 2}\right)$. The complexity $(T)$ of the processes of creating, modifying and using geoportal systems is based on the modularity of the framework $\left(T_{G I}\right)$, high modifiability of components $\left(T_{G 2}\right)$, maintainability of a software project $\left(T_{G 3}\right)$.

The introduction of effective geoportal technologies and the development of new architectural patterns based on modern web technologies and behavioral optimization makes it possible to organize a fundamental basis for obtaining and system analysis of expert information, forecasting natural processes, providing the foundations for sustainable development of territories.

4) External components and SDI actors are include users and providers of geospatial data and actors interacting with the case law of the system.

From the point of view of the set-theoretic representation, we will give a formalized description of the platform architecture of project-oriented SDIs (Fig. 1). For this, we represent the object of research as a system consisting of a set of interacting elements.

$S=\langle C, R, Q, X\rangle$

where $S$ - project-oriented SDI as a system, $C$ - a set of key components into which the project-oriented SDI is decomposed, which, based on the formulated hypothesis, can be represented as $\left\langle C_{\text {analysis }}, C_{\text {storage }}, C_{\text {geoportal }}\right\rangle$ where $C_{\text {analysis }}-$ spatial data analysis system, $C_{\text {storage }}$ - cloud storage centers for spatial data, $C_{\text {geoportal }}$ - geoportal system; $R$ - a set of relationships between SDI components, determined by the peculiarities of technical and regulatory interaction; $Q-$ set of properties of key components of SDI and relations $\left\langle Q_{C}, Q_{R}\right\rangle$, determining the criteria for optimizing spatial data; $X-$ a set of actions with the actors of the system. As the SDI develops, the set-theoretic formula can be refined to reflect the interactions between sets of components. 


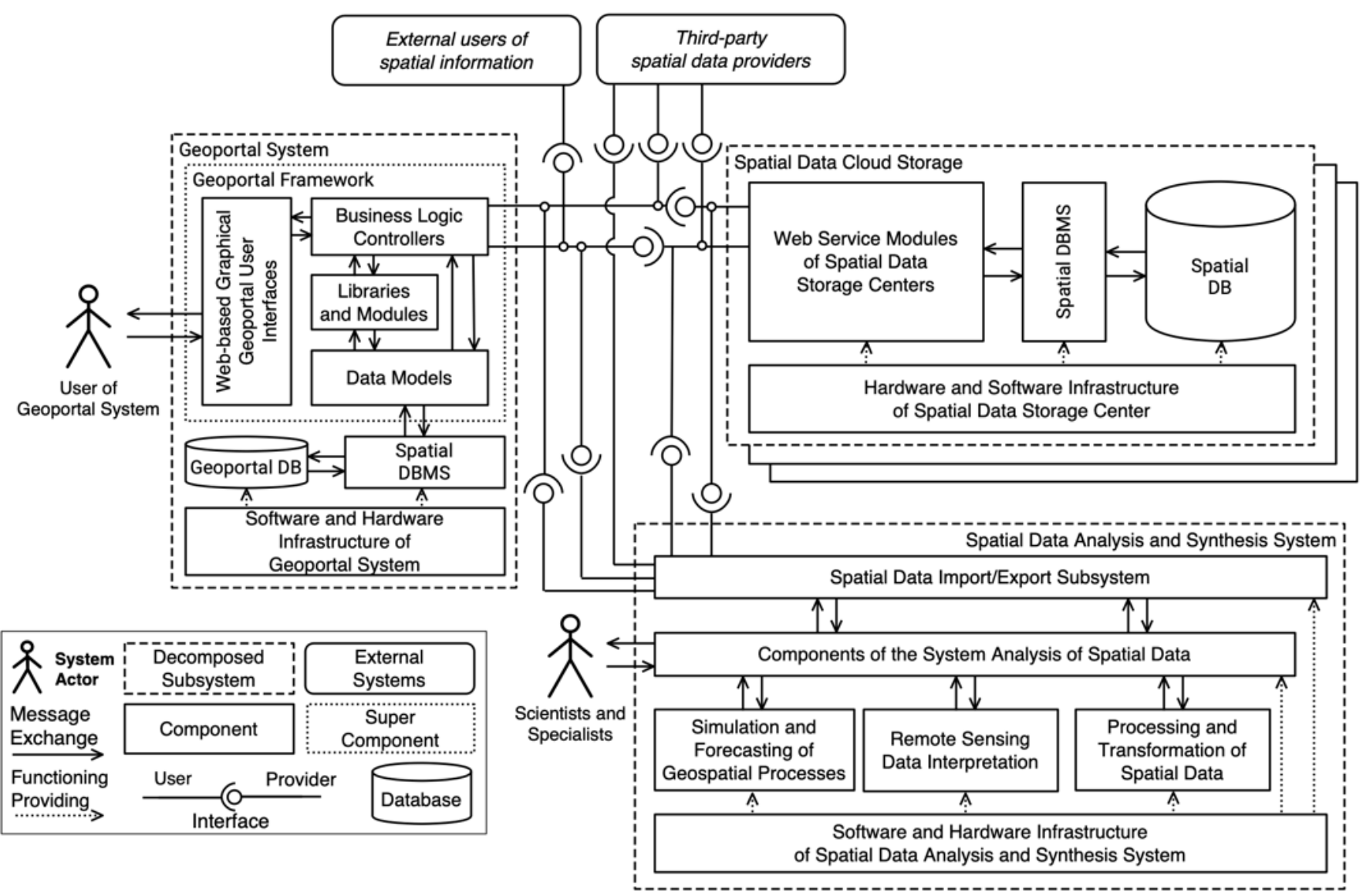

Fig. 1. Structural Component Scheme of Spatial Data Infrastructure.

In order to optimize the use and storage of spatial data, it is necessary to develop criteria for the effectiveness of the projected SDI, defined as follows:

$P=\langle E, \Re, T\rangle$

where $P$ - performance indicators of the project-oriented SDI; $E$ - set of target effects of the system, determined by specific aspects of SDI application; $\mathfrak{R}$ - resource intensity of
SDI when performing operations of storage, analysis, distribution and visualization of spatial data; $T$ - the complexity (including time) of the processes of building a system, its modification, reorganization and practical use.

Efficiency criteria of a project-oriented SDI for analysis subsystems, data storage and geoportals in order to achieve target effects, reduce resource intensity and time complexity of the system transformation are summarized in Table I.

TABLE I. CRITERIA FOR THE EFFECTIVENESS OF A PROJECT-ORIENTED SDI

\begin{tabular}{|c|c|c|c|}
\hline \multirow{2}{*}{$\begin{array}{l}\text { Performance } \\
\text { indicator }\end{array}$} & \multicolumn{3}{|l|}{ SDI subsystems } \\
\hline & $S A$ & $S D S C$ & Geoportals \\
\hline $\begin{array}{l}\text { Achievability of target effects } \\
(E)\end{array}$ & $\begin{array}{l}\text { Objective metrics improving } \\
\left(E_{A 1}\right) \text {, reduction of type I and II } \\
\text { errors }\left(E_{A 2}\right) \text {, optimization of } \\
\text { expert assessments }\left(E_{A 3}\right)\end{array}$ & $\begin{array}{l}\text { Complexity and completeness }\left(E_{S I}\right) \text {, } \\
\text { relevance, reliability and usefulness }\left(E_{S 2}\right) \text {, } \\
\text { security }\left(E_{S 3}\right) \text { of spatial data }\end{array}$ & $\begin{array}{l}\text { Functional suitability and design } \\
\text { orientation }\left(E_{G 1}\right) \text {, usability and reliability } \\
\left(E_{G 2}\right) \text {, security of user data }\left(E_{G 3}\right)\end{array}$ \\
\hline $\begin{array}{l}\text { Resource intensity }(\Re) \\
\text { of SDI }\end{array}$ & $\begin{array}{l}\text { Algorithm optimization }\left(\mathfrak{R}_{A I}\right) \text {, } \\
\text { increase in hardware power }\left(\mathfrak{R}_{A 2}\right)\end{array}$ & $\begin{array}{l}\text { Indexing records }\left(\Re_{S I}\right) \text {, optimization and } \\
\text { caching of requests }\left(\Re_{S 2}\right) \text {, development of } \\
\text { search algorithms }\left(\mathfrak{R}_{S 3}\right)\end{array}$ & $\begin{array}{l}\text { Software optimization of the geoportal } \\
\text { framework }\left(\Re_{G 1}\right) \text {, increase in dedicated } \\
\text { server resources }\left(\Re_{G 2}\right)\end{array}$ \\
\hline $\begin{array}{l}\text { Complexity }(T) \text { of the processes } \\
\text { of creating, modifying and using } \\
\text { a component }\end{array}$ & $\begin{array}{l}\text { Modularity of the model }\left(T_{A 1}\right) \text {, } \\
\text { optimization of software metrics } \\
\left(T_{A 2}\right) \text {, testability }\left(T_{A 3}\right)\end{array}$ & $\begin{array}{l}\text { Standardization and documentation }\left(T_{S 1}\right) \\
\text { integrity }\left(T_{S 2}\right) \text {, normalization }\left(T_{S 3}\right) \text { of } \\
\text { database }\end{array}$ & $\begin{array}{l}\text { Modularity of the framework } \\
\left(T_{G I}\right) \text {, high modifiability of components } \\
\left(T_{G 2}\right) \text {, project maintainability }\left(T_{G 3}\right)\end{array}$ \\
\hline
\end{tabular}


Let us formulate the supporting points that are critical in the issue of optimizing the use and storage of geospatial data:

1) Improvement the accuracy and speed of methodological, algorithmic and software for the process of analyzing spatial data and predicting space-time processes.

2) Improvement of storage systems for large spatiotemporal data through the development of new physical and logical storage models.

3) Implementation of effective geoportal technologies and development of new architectural patterns of spatio-temporal data using modern web technologies.

Achievement of a comprehensive solution of reference problems will make it possible to obtain a system of effective methods, algorithms and architectural patterns for storage, operational analysis and use of spatial data for the construction of design-oriented SDI.

\section{Algorithmic Support of the Process of ANAlysis of Spatial Data}

The development of new geographic information algorithms and methods for analysing spatio-temporal data for analysing land parcels and predicting emergencies is now of great relevance. Evaluation and drawing conclusions based on in-depth analysis of spatial and temporal data are used for various practical tasks, such as environmental monitoring, disaster prediction. Deep machine learning can reduce research costs by allowing accurate interpolation and extrapolation of measurements. In the problem area under analysis, the largest number of publications are devoted to neural networks, for which networks with a multilayer system of non-linear filters are used to extract hierarchical features from the data [1].

The key to improving the accuracy of in-depth analysis of spatial data lies not only in improving the architectures of deep machine learning models, but also in developing efficient data processing [2]. The problem should be addressed from a geosystemic perspective, using the hypothesis of deep relationships of geographical elements [3]. From this standpoint lower level of the hierarchy constituting the territory to be analyzed. On this basis, it can be assumed that the accuracy of the classification of geosystems based on remotely sensed data can be improved if the nomenclature takes into account only the properties of a particular territory, but also the characteristics of the geosystems to which it belongs.

Next, provide a description of the data model that allows us to characterize the territory in terms of the geosystem approach, with a view to subsequently classifying the geosystems using machine learning models that can effectively analyze the data [4].

By classification we mean the operation $\mathrm{f}$ executed by the model $M$, with the experience $E$. This allows us to correlate a class $y$ specific label with the $\mathrm{x}_{\text {Local }}$ parameter and in a direct relationship with geosystems and the $\mathrm{x}_{\mathrm{Geosystem}}$ property vector:

$y=f\left(\left\langle\mathrm{x}_{\text {Local }}, \mathrm{X}_{\text {Geosystem }}\right\rangle, M, E\right)$

If $\mathrm{X}_{\text {Geosystem }}$ is empty, we consider the case of classification without involving geodata. Thus, a territory can be assigned to a class based on pixel-based classification or by extracting features from territorial fragments of different sizes (patchbased classification). From a geosystem approach, the properties of a territory are highly dependent on a geosystem. Data from remote sensing of the Earth serve as a source of information. But if strict rules are presented to $\mathrm{L}_{0}$ level data about $\mathrm{x}_{\text {Geosystem }}$ object, they must be obtained in a strictly necessary period and of high quality.

The fact that these data have low temporal resolution is the reason for their low price. At the same time, they do not cease to be an informative source of $\mathrm{x}_{\text {Geosystem }}$ on closed geosystems. Obtaining data from levels $L_{1}, L_{2}, \ldots, L_{N}$ can potentially be automated: it has information about the geographic latitude and longitude of the terrain and it is possible to request a fragment of a space image of the territory with these coordinates of required scale and resolution.

Let us hypothesize that electronic landscape maps and other geographical maps (land cover, land use) are traditionally the ultimate artifact of the process of analysing and classifying remote sensing data, have a substantial amount of information about the territories included in them and form input tensors in the $\mathrm{x}_{\text {Geosystem }}$ set. The high degree of abstraction indicates that maps have a good potential to enrich information on small classified areas within the geosystems.

To classify information on the geosystem model of the territory, presented in the form of a tuple $\left\langle\mathrm{x}_{\mathrm{Local}}, \mathrm{x}_{\mathrm{Geosystem}}\right\rangle$, a deep neural network was proposed that accepts data tensors and returning the hypothesis that a given territory belongs to a certain class. This model can be used both separately and in an ensemble with other mono-classifiers.

From the point of view of the black box, the classifier considered above obtains on the inlet images of the territory $\left(\mathrm{L}_{0}\right)$ and contains geosystems $\left(\mathrm{L}_{\mathrm{i}}\right)$ obtained from satellite images as well as synthetic maps ( $\left.L_{s}\right)$. Their number may vary depending on the territorial model levels, but their growth should be treated with caution, as this will inevitably lead to an increase in the capacity of the model.

The next layer is the batch normalization layer, which allows achieving regularization and model stability. At the end of the feature extraction block is a subdecritization layer that applies the maximum operation to reduce the size of the resulting representations and has external outputs. The next component block of the described model is the feature fusion module. It accepts as input the N-level features extracted from the images of the classified territory. Also, the second and subsequent level merge modules accept the output of the previous merge module as input.

The output layer of the merger module of the last feature is transformed into a vector and fed to the input of the multilayer perceptron. The number and thickness of the interconnected layers are selected on the principle of minimizing these parameters while maintaining sufficient accuracy in the classification. Deep models may examine more signs, but they are very susceptible to problems of retraining [5].

At the same time, an important place is occupied by the problem of adaptation of individual classifiers 
(monoclassifiers) to a new set of data. It is possible to approach the solution of the indicated problems by combining individual classifiers into ensembles. Research results show that the combination of classifiers into a system improves the stability of the classification algorithm.

The key system components of the ensemble of classifiers can be a set of individual monoclassifiers of various architectural organizations and a metaclassifier - a module that receives data from monoclassifiers for the subsequent adoption of the resulting decision on the belonging of the analyzed spatial territorial object to any class. Let us give a description of the algorithm that determines the variant of ensemble training, according to which the following tasks are sequentially solved: 1) training of individual monoclassifiers, 2) assessment of the accuracy of each individual monoclassifier; 3) training the metaclassifier that analyzes the decisions made by the mono-classifiers of the ensemble, taking into account weighted voting:

$\left\{\begin{array}{c}H=\operatorname{argmax}_{c \in C}\left(\sum_{i=1}^{N} w(i, c) V(i, c)\right), \\ V(i, c)=1, \text { if } i-\text { th classifier defines class } c, \\ V(i, c)=0, \text { if } i-\text { th classifier rejects class } c,\end{array}\right.$

where $w(i, c)$ is a weight coefficient characterizing the efficiency of the $i$-th classifier in detecting territorial objects of class c from the general set of classes $C ; V(i, c)$ is a boolean variable describing the fact that the $i$-th classifier classified the territorial object as class $c ; N$ is the total number of ensemble monoclassifiers.

The $F_{\beta}$-score metric can be used as a weighting coefficient of efficiency, which is a value that depends on accuracy and sensitivity.

$$
\begin{aligned}
& F_{\beta c}(i)=\left(1+\beta^{2}\right) \frac{\text { precision } \cdot \text { recall }}{\left(\beta^{2} \cdot \text { precision }\right)+\text { recall }}= \\
& =\frac{\left(1+\beta^{2}\right) T P_{c}(i)}{\left(1+\beta^{2}\right) T P_{c}(i)+\beta^{2} F N_{c}(i)+F P_{c}(i)}
\end{aligned}
$$

where $T P_{c}(i)$ is the number of correctly classified territorial objects of class c; $F P_{c}(i)$ - type I error for territorial objects of class $c ; F N_{c}(i)$ - type II error for territorial objects of class $c$.

If we take the value $\beta=1$, then the metric will take the value of the harmonic mean of sensitivity and accuracy (Fscore). The methodology for calculating the efficiency ratio can be developed by introducing the concept of an inefficiency threshold. At the same time, the formula for calculating the weight coefficient for the $i$-th classifier of the ensemble, typical for the definition of territorial objects of class $c$, will take the following form:

$w(i, c)=\left\{\begin{array}{c}0, \text { if } G_{\mathrm{c}}(i)-\varepsilon \leq 0, \\ \frac{1}{1-\varepsilon}\left(F_{\mathrm{c}}(i)-\varepsilon\right), \text { if } F_{\mathrm{c}}(i)-\varepsilon>0\end{array}\right.$

where $\varepsilon$-inefficiency threshold.

The value of the proposed weighting metric will be equal to one in the case of an ideal classifier and zero if the quality of the classification goes beyond the inefficiency threshold $\varepsilon$. In the general case, the presented efficiency metric, which depends on sensitivity and accuracy, determines the model's ability to correctly classify objects of a particular territorial class, avoiding a high number of type I and II errors. If the parameter $\varepsilon$ is taken equal to 0.5, the hypotheses of monoclassifiers will be discarded, which gives a result with a simple guessing accuracy.

The process of finding an effective classification of a mono-model can be represented as a decision tree, the main node of which precedes the first stage of the search algorithm, corresponds to the solution of the problem of formulating a study problem. The nodes of the tree define a variant of fixing the state of the model at the $i$-th stage of the algorithm for finding an effective model.

\section{INTEGRATION OF SPATIAL DATA}

As noted in the previous section, geosystems are systems that are equally geographic, economic, social and technical, including a variety of ecological, social, economic objects, processes, phenomena and their interconnections. It is recommended that basic mapping support be defined a focused SDI as a repository of geographical maps and images for storage in a database and subsequent use to improve the performance of natural and social production systems.

The landscape envelope and the hierarchy of geosystems are the arena for the development of global, regional and local processes of development. In the author's interpretation, a relationship-relationship is any form of correspondence of phenomena, which can be represented in the form of a functional dependence of variable quantities, which has either an unambiguous character or a probabilistic one, and a relationship-interaction is a process of mutual interaction of objects that occurs in real space and time and expressed in a certain course of events that occur with matter and energy in a certain area of space.

Diagnostics and assessment of relationships in the geosystem hierarchy is an important area in the development and practical use of new automated geo-data processing algorithms and supporting space-based temporal formation using machine learning within SDI, continuous updating of cloud storages of large spatial and temporal data for organizations for which the multi-model approach is relevant $[6,7]$. The solution to this problem is achieved through the introduction of geoportal systems, which are characterized by a project orientation and are an indisputable basis for the work of state and municipal bodies, which operate strategic sectors of the economy $[8,9]$.

As a result, it can be argued that in SDI, in order to assess the functioning of natural and social production systems, it is appropriate to distinguish between the following levels of data integration and use:

1) The level of basic spatio-temporal data, including the basic cartographic basis and describing the modern and historical spatio-temporal structure of the geographic envelope, the patterns of interaction of geospheres.

2) The level of spatial models assumes the systematization of data according to the following modules: "natural systems", "social systems", "economic systems", "geoecological systems". 
3) The level of decision-making presupposes a projectoriented use of data for organizing the processes of geographical forecasting (and the formation of a complex characteristic of countries and regions (systematizing geodata).

Let us formulate the key principles of spatial data integration from the perspective of the geosystem approach:

1) Modern digital methods for analyzing spatial information form the basis for the study of geosystems representing a hierarchical model of the territory. This technology is promising, as it enhances SDI capabilities.

2) Analysis is enhanced by the use of multiple data warehouses. This provides a broad perspective on the use of spatial data for effective management decisions.

3) No single paradigm the storage of spatio-temporal information in SDI can provide a key to solving all problems, due to the need for different representations for different types of spatio-temporal data. The answer to the problem of integration, storage and processing of large arrays of spatiotemporal information in digital SDIs can be provided by multimodel storages, hybrid databases based on the functionality of different classes of DBMS.

4) The consequence of the use of multi-model storages is the strengthening of the qualitative characteristics of the digital SDI - flexible scaling of the storage as needed, increasing the reliability and fault tolerance.

Spatial data can be stored in data centres or cloud warehouses.

\section{IMPLEMENTATION AND PROJECT-ORIENTED USE OF GEOPORTAL SYSTEMS}

In the context of the rapid information and economic growth of the regions, the development and use of geoorthographic systems (GIS) plays an important role. Such systems provide access to information on the natural resources, economic and social situation of the regions [10]. Geoportals provide information retrieval and monitoring through integrated research.

Geographic Information Systems, which have inherited the main features of a large number of software systems of a wide range of software information systems, traditionally with high functional interface requirements, with whom they interact with the outside world. These include the Application Programming Interface (API) and the Graphical User Interface (GUI). The first type of interfaces interacts automatically with GIS for data exchange [11]. The GeoJSON, TopoJSON and KML data formats are used to increase the efficiency of this process. In order to make effective use of GIS, it is necessary to develop a reliable and easily modifiable programming interface $[12,13]$.

A review of previously developed geoport solutions highlighted the following key points for an effective interface: 1) focus on flexible development; 2) use of cross-platform web interfaces; 3) focus on future developments; 4) move away from GIS resource management systems; 5) use of necessary libraries; 6) focus on problem solving and achieving objectives.
The main functional requirements for geoorthographic systems are as follows: availability of cartographic data, possibility of combining layers, use of convenient navigational tools, access to the properties of spatial data. Among the many important qualitative requirements are: adaptive GUI for different devices, user-friendly interfaces, portability and extensibility, data security and system reliability.

Let's move on to considering the solutions implemented by the authors of this article, applying the qualitative and functional requirements described above. In order to visualize and disseminate information about the cultural heritage of the landscape of the Republic of Mordovia, a geoportal system "Natural and Cultural Heritage of Mordovia" has been created, accessible at https://tourismportal.net/. The system discloses information about the natural, socio-cultural and infrastructural potential of the region. The portal interface is characterized by its properties of comprehensibility, adaptability, scalability and security.

Several other features of geoportal systems development are implemented in the Lifeshot.info project. The emphasis is on the use of user-friendly cartographic interfaces with news information rather than geospatial information, as is traditionally the case. Thus, more attention is being paid to the cartographic base. The control components receive up-to-date information about news and events occurring in the vicinity of the user. This approach is unique and different from other geoportal and news environments. Scientific novelty is achieved by combining two types of systems.

\section{CONCLUSION}

A set-theoretic approach to the analysis of system properties, elements and their connections of design-oriented SDIs makes it possible to single out the supporting points that are critical in optimizing the storage processes and the subsequent use of spatio-temporal data and identify ways for further research:

1) Improving the accuracy of methodological, algorithmic and software for the process of analyzing spatial data and predicting space-time processes. The solution to this problem is possible through the development and use of new algorithms for deep machine analysis for the complex interpretation of remote sensing data, digital synthetic landscape maps and auxiliary spatio-temporal information in order to carry out high-precision automated monitoring of the state of ecologicalsocio-economic systems and forecasting natural-man-made emergencies.

2) Improving storage systems for large spatio-temporal data by developing multi-model approach, synthesizing relational and NoSQL paradigms, introducing new algorithms for caching and indexing large information arrays, creating methods for storing and processing large amounts of information using cloud technologies.

3) Implementation of effective geoportal technologies and development of new architectural patterns for visualization and dissemination of spatio-temporal data using modern web technologies and behavioral optimization in order to organize a 
fundamental basis for obtaining and systematic analysis of expert information when making management decisions.

Achieving a comprehensive solution to reference problems allows you to obtain a system of effective methods, algorithms and architectural patterns for storage, operational analysis and dissemination of spatio-temporal data for the construction of project-oriented SDI.

\section{ACKNOWLEDGMENT}

The reported study was funded by RFBR, project number 20-37-70055.

\section{REFERENCES}

[1] E. O. Yamashkina, S. M. Kovalenko, O. V. Platonova, "Development of repository of deep neural networks for the analysis of geospatial data," in IOP Conference Series: Materials Science and Engineering, vol. 1047, no 1, 012124, Feb. 2021. DOI: http://dx.doi.org/10.1088/1757-899X/1047/1/012124.

[2] J. V. Alva, "Neural Networks with the Wolfram Language," in Beginning Mathematica and Wolfram for Data Science, pp. 331-373, 2021.

[3] L. Miklós, E. Kočická, Z. Izakovičová, D. Kočický, A. Špinerová, A Diviaková, V. Miklósová, Landscape as a geosystem, Springer, Cham, 2019, pp. 11-42.

[4] S. A. Yamashkin, A. A. Yamashkin, V. V. Zanozin, M. M Radovanovic, A. N. Barmin, "Improving the Efficiency of Deep Learning Methods in Remote Sensing Data Analysis: Geosystem Approach," IEEE Access, vol. 8, pp. 179516-179529, Oct. 2020. DOI: http://dx.doi.org/ 10.1088/1757-899X/1047/1/012124.

[5] G. Cheng, C. Yang, X. Yao, L. Guo, J. Han, "When deep learning meets metric learning: Remote sensing image scene classification via learning discriminative CNNs," IEEE Transactions on Geoscience and Remote Sensing, vol. 56, no. 5, pp. 2811-2821, Jan. 2018. DOI: http://dx.doi.org/ 10.1109/TGRS.2017.2783902.

[6] J. Lu, I. Holubová, "Multi-model databases: a new journey to handle the variety of data," ACM Computing Surveys (CSUR), vol. 52, no. 3, pp. 1-38, Jun. 2019. DOI: http://dx.doi.org/10.1145/3323214.

[7] J. G. Lee, M. Kang, "Geospatial big data: challenges and opportunities," Big Data Research, vol. 2, no. 2, pp. 74-81, Feb. 2015. DOI: http://dx.doi.org/10.1016/j.bdr.2015.01.003.

[8] R. Taft, I. Sharif, A. Matei, N. VanBenschoten, J. Lewis, T. Grieger, P. Mattis, "Cockroachdb: The resilient geo-distributed SQL database," in Proceedings of the 2020 ACM SIGMOD International Conference on Management of Data, pp. 1493-1509, Jun. 2020.

[9] J. Han, E. Haihong, G. Le, J. Du, "Survey on NoSQL database," in 2011 6 th international conference on pervasive computing and applications, pp. 363-366, Oct. 2011.

[10] M. Li, W. Guo, L. Duan, X. Zhu, "A case-based reasoning approach for task-driven spatial-temporally aware geospatial data discovery through geoportals. International Journal of Digital Earth," vol. 10, no. 11, pp. 1146-1165, Jun. $2017 . \quad$ DOI: http://dx.doi.org/10.13203/j.whugis20140823.

[11] C. Granell, I. Miralles, L. E. Rodríguez-Pupo, A. González-Pérez, S. Casteleyn, L. Busetto, J. Huerta, "Conceptual architecture and serviceoriented implementation of a regional geoportal for rice monitoring," ISPRS International Journal of Geo-Information, vol. 6, no. 7, 191, Jun. 2017. DOI: http://dx.doi.org/10.3390/ijgi6070191.

[12] S. A. Yamashkin, M. M. Radovanović, A. A. Yamashkin, A. N. Barmin, V. V. Zanozin, M. D. Petrović, "Problems of designing geoportal interfaces," GeoJournal of Tourism and Geosites, vol. 24, no. 1, pp. 88101, Jan. 2019. DOI: http://dx.doi.org/10.30892/gtg.24108-345.

[13] S. Yeruva, M. S. Varalakshmi, B. P. Gowtham, Y. H. Chandana, P. K. Prasad, "Identification of Sickle Cell Anemia Using Deep Neural Networks," Emerging Science Journal, vol. 5, no. 2, pp. 200-210, Apr. 2021. 